\title{
Interstitial Deletion of 5q22.2q23.1 Including APC and TSSK1B in a Patient with Adenomatous Polyposis and Asthenoteratozoospermia
}

\author{
Tanya Kadiyska $^{a, c}$ Ivan Tourtourikov ${ }^{a}$ Asen Petrov $^{b}$ Ani Chavoushian ${ }^{b}$ \\ Miglena Antalavicheva $^{b}$ Eva-Maria König ${ }^{d}$ Eva Klopocki $^{d} \quad$ Nikolova Vessela ${ }^{a}$ \\ Romil Stanislavov ${ }^{a}$ \\ ${ }^{a}$ Genetic Medico-Diagnostic Laboratory Genica, ${ }^{b}$ Department of Gastroenterology, City Clinic Cancer Center, and \\ 'Department of Medical Chemistry and Biochemistry, Sofia Medical University, Sofia, Bulgaria; ${ }^{\mathrm{d}}$ Institute of Human \\ Genetics, Biozentrum, University of Würzburg, Würzburg, Germany
}

\section{Established Facts}

- Interstitial 5q22 deletions are rare and typically accompanied by neurological and developmental complications.

- Most genes within the chromosomal band 5q22 have not been extensively studied nor associated with a clinical phenotype.

\section{Novel Insights}

- We present a case with a heterozygous deletion in the $5 q 22.2 q 23.1$ locus that does not exhibit the developmental issues that typically arise from this type of deletion.

- The TSSK1B gene within this locus has recently been functionally tested in model organisms; here we can see the first report of haploinsufficiency of this gene potentially causing a human phenotype.

- TSSK1B can be a putative cause for male factor infertility.

\section{Keywords}

Array $\mathrm{CGH} \cdot$ Asthenoteratozoospermia · Familial adenomatous polyposis $\cdot$ TSSK1B

\begin{abstract}
Interstitial 5q22 deletions are relatively rare and usually represented by severe clinical features such as developmental delay and growth retardation. Here, we report a 23-year-old male patient, referred to our laboratory for genetic confirmation of possible familial adenomatous polyposis. MLPA and the subsequent array CGH identified an approximately
\end{abstract}

8-Mb-sized deletion in the 5q22.2q23.1 locus. Further analysis of the deleted region and the genes within suggested a possible role for the TSSK1B (testis-specific serine/threonine kinase 1) gene in the patient's reproductive capacity. Semen analysis confirmed that the patient's reproductive capability was impaired, and that he suffered from asthenoteratozoospermia. Analysis of the azoospermia factor region on the $Y$ chromosome revealed no microdeletions. Further sequencing tests could not find an alternative explanation for the patient's infertility. This case demonstrates a possible role of TSSK1B in male reproduction.

C 2018 S. Karger AG, Basel

\section{KARGER}

(C) 2018 S. Karger AG, Basel

E-Mail karger@karger.com

www.karger.com/msy
Tanya Kadiyska, $\mathrm{PhD}$

Department of Medical Chemistry and Biochemistry

Sofia Medical University

2 Zdrave St., BG-1431 Sofia (Bulgaria)

E-Mail kadiyska_t@yahoo.com 
Interstitial $5 \mathrm{q} 22 \mathrm{q} 23$ deletions are relatively rare and usually accompanied by severe clinical features such as developmental delay, growth retardation, and dysmorphic features [Bennett et al., 1997]. Deletions in this region often involve the $A P C$ tumor suppressor gene (OMIM 611731) and lead to familial adenomatous polyposis (FAP). FAP is an inherited autosomal dominant condition leading to multiple intestinal polyps and predisposition to colon cancer. Although the first reported 5q interstitial deletion was in 1979 [Kadotani et al., 1979], the APC locus was discovered later by Kinzler et al. [1991], who mapped it to the $5 \mathrm{q} 22.2$ band. Because of the timing of this discovery, all the clinical cases of $5 q$ deletions up to that moment did not examine the manifestation of FAP in the patient, and therefore do not contribute any information about the link between 5q22 deletions and FAP. Patients with chromosomal deletions that include $A P C$ are most often diagnosed when their dysmorphic features are revealed in infancy or when they fail to thrive and show other signs of delayed development [Lee et al., 2012; Yamaguchi et al., 2014]; those that remain asymptomatic usually experience gastrointestinal manifestations such as weight loss, rectorhaggia and bowel obstruction.

Because of the thorough characterization of the molecular basis for FAP and the severity of its pathological symptoms, clinical focus is most often concentrated on the APC gene itself or physical consequences of a large chromosomal deletion, revealing little to no information about the function of other genes in the $5 \mathrm{q} 22.2$ band. Although a number of chromosomal aberrations have been described [Bennett et al., 1997; Tzschach et al., 2006, Heald et al., 2007; Casper et al., 2014], analysis of the consequences tends to focus on the resulting dysmorphic features or FAP without imparting function to single genes. Large deletions within the chromosome itself present with a complex phenotype, combining several clinical features from those mentioned above and can be a challenge for the diagnosing clinician.

\section{Case Report and Methods}

The patient is a 23-year-old Bulgarian male without prior clinical history. Upon his first admission, he was complaining of frequent rectorhaggia with no abnormalities in defecation frequency or loss of appetite. A sigmoidoscopy was performed with results pointing towards FAP, for which he was directed to a specialized gastroenterological department for further testing. Family history was unavailable due to ethical reasons, as the patient had been in a foster family since an early age, and there was no
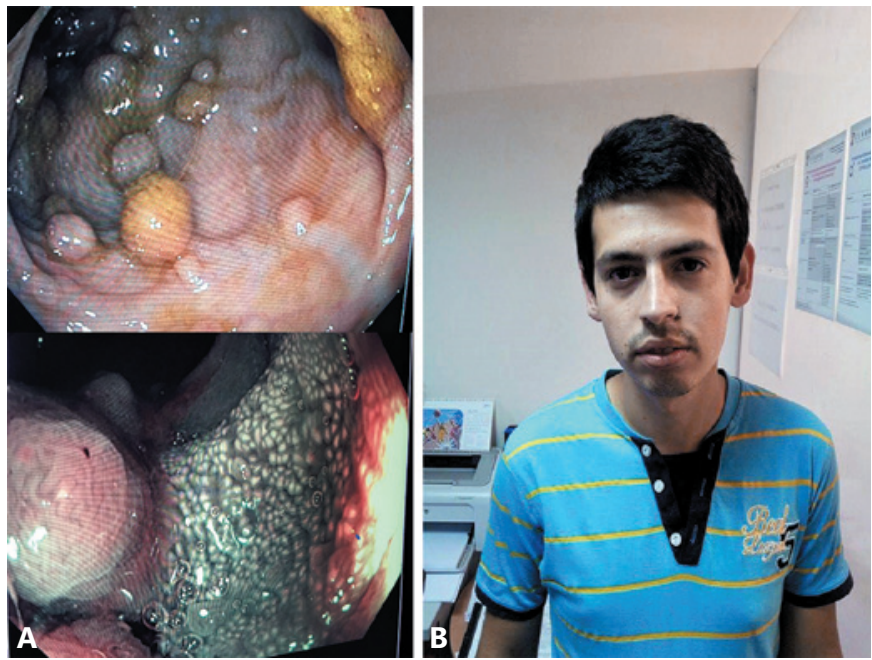

Fig. 1. A Fibrocolonoscopy images showing different polyps. B The proband, a 23-year-old Bulgarian male, with no discernible dysmorphic features.

contact with his biological parents. Fibrocolonoscopy revealed a large number of polyps in the rectum and the sigmoid - pale to dark red - with hyperplasia (Fig. 1A). There were no FAP-associated lesions outside of the gastrointestinal tract. At this point, the patient was referred to our genetic laboratory to confirm the FAP diagnosis.

Genomic DNA was isolated from peripheral blood using standard protocols and was used to screen the $A P C$ gene for larger deletions and duplications using MLPA. The MLPA APC kit was obtained from MRC-Holland (PO43, Amsterdam, The Netherlands), and analysis was performed according to the manufacturer's instructions.

In order to determine the boundaries of the deletion, genomic DNA was analyzed by array CGH on a high-resolution 1M oligonucleotide array according to the manufacturer's protocol (Agilent Technologies, Santa Clara, CA, USA). Copy number variation $(\mathrm{CNV})$ detection was conducted using CytoGenomics software (version 2.9.2.4, Agilent Technologies). The following analysis settings were used: aberration algorithm ADM-2, threshold: 6.0 , window size: $0.5 \mathrm{Mb}$, filter: 5 probes, and $\log _{2}$ ratio $=0.29$.

Ejaculate was detached in the laboratory and studied in the order: observation of native preparation, determining sperm concentration, assessment of swabs for vitality in mobility $<40 \%$, and evaluating sperm morphology according the criteria of ESHRE/ NAFA, using Sperm Class Analyzer (SCA), a system of quantitative and qualitative analysis of parameters of human sperm.

Molecular testing for microdeletions in the azoospermia factor region were performed as described in the guidelines and standards of the European Academy of Andrology [Krausz et al., 2014].

Sanger sequencing was performed to examine previously reported regions associated with male infertility, i.e., DAZL (OMIM 601486), SEPT12 (OMIM 611562), SLC26A8 (OMIM 608480), SYCE1 (OMIM 611486), and subsequently, TSSK1B (OMIM 610709). 


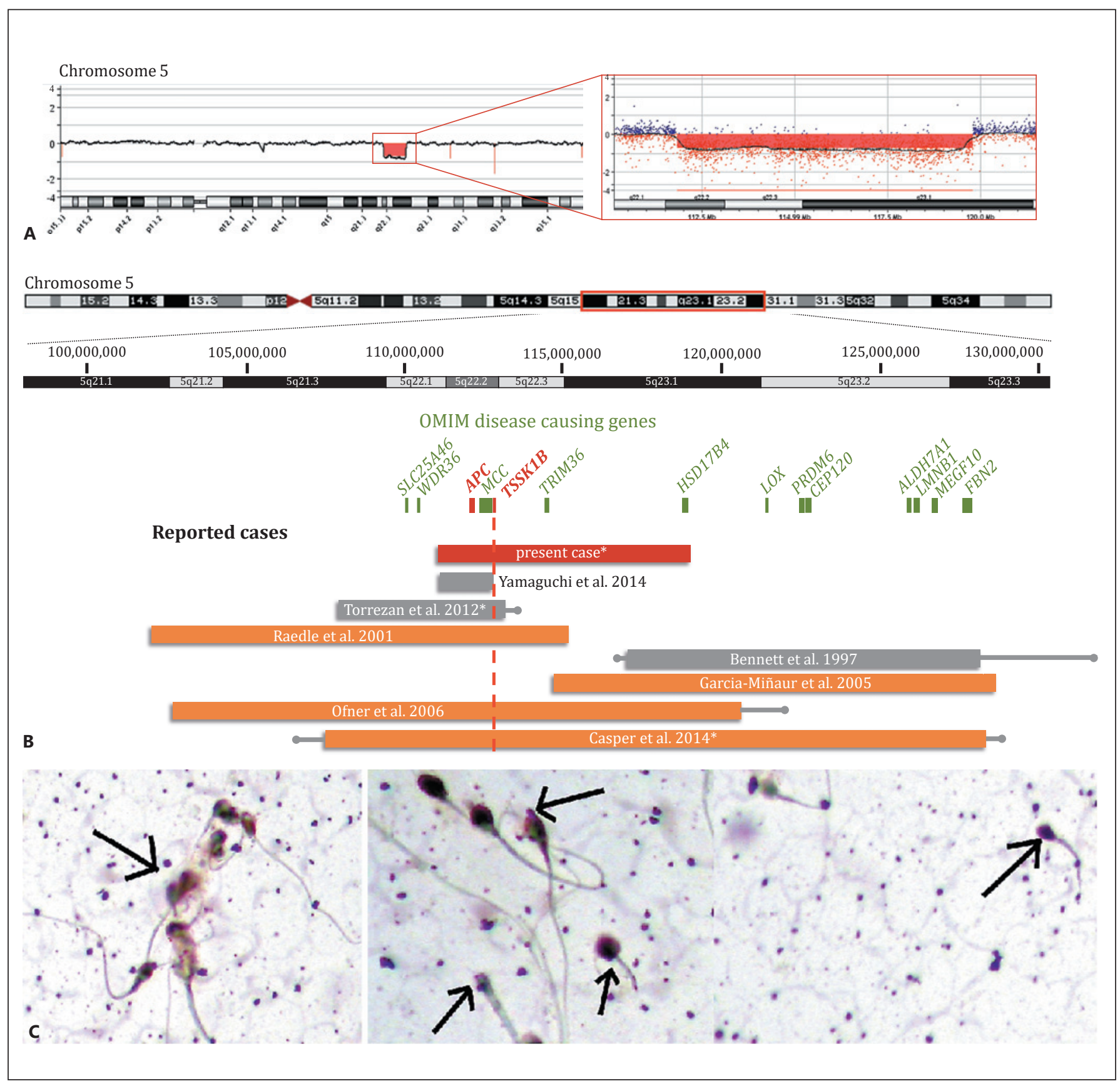

Fig. 2. Array-CGH analysis, genomic overview of the chromosomal region 5q21.1q23.3, and sperm defects observed in our patient. A Array-CGH profile of chromosome 5 shows a $5 \mathrm{q}$ interstitial deletion. The 8-Mb 5q22.2q23.1 deletion (indicated by the red bar) is shown in greater detail. B Disease-causing OMIM genes are depicted as green bars within the overview of the genomic region encompassing the cytobands $5 \mathrm{q} 21.1$ to $5 \mathrm{q} 23.3$. The genes $A P C$ and $T S S K 1 B$ are highlighted in red. In the lower part, the $8-\mathrm{Mb}$ deletion of our patient is compared with overlapping deletions of patients reported in the literature (all positions according to GRCh37/ hg19). Note the case from Yamaguchi et al. [2014] does not encompass the TSSK1B gene. The cases indicated in orange present with severe developmental delay and/or dysmorphic features. The asterisks mark cases analyzed by array CGH. C Spermatozoa with pointed heads, a round-headed sperm with a short tail, and decapitated sperm as well as microcephalic sperm and a sperm head without DNA content (arrows).
Interstitial Deletion of 5q22.2q23.1

Including $A P C$ and TSSK $1 B$
Mol Syndromol 2018;9:235-240

DOI: $10.1159 / 000492516$ 


\section{Results}

We found a $50 \%$ peak area decrease in all $A P C$-specific gene probes by MLPA. As this corresponded to a heterozygous deletion of the entire $A P C$ gene sequence, our next step was to perform an array CGH to determine the boundaries of the deletion. The obtained results showed that the interstitial deletion in chromosome $5 \mathrm{q}$ was confined to the region 5q22.2q23.1, approximately $8 \mathrm{Mb}$ long (Fig. 2A). The deletion spans the region between base pairs chr5: 111,779,298-119,794,955 (GRCh37/hg19), karyotype: arr[hg19] 5q22.2q23.1(111779298_119794955) ×1 [ISCN 2016], confirming the APC deletion and involving 29 other protein-coding genes in the deletion. Along with the well-described $A P C$ and MCC genes, another gene, TSSK1B, had been previously studied and attributed with a specific function. TSSK1B is highly expressed in testis and required during spermatid development [Hao et al., 2004]. Therefore, we decided to perform a semen analysis and test our patient for infertility, sperm count, morphology, and motility.

The final evaluation concluded that the patient suffered from asthenoteratozoospermia with a prevalence of cephalic defects, 97\% atypical sperm morphology, and reduced sperm motility (Fig. 2C). The teratozoospermia index value at 1.71 shows more than 1 defect per single sperm, pointing towards impaired spermatogenesis. Since this phenotype is most often caused by microdeletions in the azoospermia factor region and large chromosomal deletions within the $\mathrm{Y}$ chromosome were not shown on the array CGH, we performed molecular testing for possible microdeletions in the $\mathrm{Y}$ chromosome. Our analysis did not reveal any deletions within the azoospermia factor markers sY84, sY86, sY127, sY134, sY254, and sY255. Testing for the most common mutations in genes associated with male infertility such as DAZL, SEPT12, SLC26A8, and SYCE1 revealed no alterations. Finally, sequencing the TSSK1B gene revealed no abnormalities within the single present copy.

These results narrowed down the possibility that TSSK $1 B$ is a strong candidate gene for male infertility and is involved in the asthenoteratozoospermia phenotype of our patient.

\section{Discussion}

Chromosomal deletions of the $5 \mathrm{q} 22$ band resulting in FAP have been rarely reported throughout the literature. Existing reports have aimed to compare and contrast de- letions between different patient cases; however, the pathological implications of those deletions have not been investigated in detail beyond the initial clinical diagnosis [Lee et al., 2012; Yamaguchi et al., 2014]. The size of the $5 q$ interstitial deleted region varies greatly among patients, ranging from 1.7 Mbp [Yamaguchi et al., 2014] to $20 \mathrm{Mbp}$ [Casper et al., 2014] and larger, with the majority of the cases presenting with various degrees of learning disability, failure to thrive, and minor to major dysmorphic signs [Rivera et al., 1990; Pilarski et al., 1999]. These symptoms are not confined to a single chromosomal band (Fig. 2B), which has prevented the classification of $5 \mathrm{q}$ deletion subtypes, although several attempts have been made [Rivera et al., 1990; Lindgren et al., 1992; Lee et al., 2012]. Notable findings from these studies show a lack of a genotype-phenotype correlation in cases that share an affected region as well as no association between the size of the deletion and the severity of the phenotype.

To the best of our knowledge, the last review of genes and their functions within this region was conducted by Ofner et al. [2006]. So far, only 4 genes (APC, MCC, TRIM36, and HSD17B4) located within the deleted region in our patient have been associated with a disease in OMIM, although research is ongoing. Furthermore, the fertility status of most patients with a $5 \mathrm{q}$ deletion was never published because of their early age, and their condition was never followed past the initial diagnosis. In previously published reports of $5 q$ deletions, only a single case [Torrezan et al., 2012] has documented a possible inheritance of a TSSK $1 B$ deletion from a suspected male carrier; however, the patient's father could not be tested to confirm that the deletion was inherited. Regarding TSSK1B encompassing CNVs in the normal population, there is only a single DGV entry of a copy loss (nsv599396, total sample size 17,421; PMID 21841781) [Cooper et al., 2011].

In the patient presented here, we did not observe severe pathological symptoms that are exhibited in typical $5 \mathrm{q} 22$ deletion cases besides FAP. This can be explained by the possibility that the deletion we present does not completely overlap with deletions in patients with severe dysmorphic/developmental issues (Fig. 2B). While the common clinical phenotype for patients with deletions in the long arm of chromosome 5 covers a wide array of features such as developmental delay, failure to thrive, psychomotor retardation, flat nasal bridge, and others [Lee et al., 2012], the phenotype presented by our patient is represented almost exclusively by the heterozygous deletion of the APC gene and the consequent FAP diagnosis.
238

Mol Syndromol 2018;9:235-240 DOI: $10.1159 / 000492516$
Kadiyska et al. 
The genetic background of testicular infertility factors, specifically asthenozoospermia, has long been limited and poorly described. The majority of testicular infertility factors and their genetic background have been linked to genes residing in the $\mathrm{Y}$ chromosome and chromosomal aberrations involving the Y chromosome [O'Flynn O'Brien et al., 2010]. There has been progress in the field, as recent research has aimed to relate sperm dysmorphology phenotypes to specific genes, such as DAZL, SYCE1, SLC26A8, SEPT12, and others that have a function related to spermatogenesis [Ray et al., 2017]. Even so, the field of male infertility genetics is still relatively new, and novel contributions are crucial to the ongoing improvement of genetic testing, diagnosis, and counseling [Lamb et al., 2016].

Testis-specific serine/threonine kinase 1 is an intronless gene, part of the TSSK family, and a branch of the serine/threonine kinase superfamily. It was first identified by Bielke et al. [1994], who in their study attributed to the gene an open reading frame of $1092 \mathrm{bp}$ coding for a 364-aa protein. Human TSSK1B was investigated using Northern and dot blots, which showed $85 \%$ similarity to murine Tssk $1 b$. The gene was mapped on chromosome $5 \mathrm{q} 22$, in a region that shows no synteny to the mouse chromosomal locus of Tssk $1 b$ [Hao et al., 2004]. Further studies have pointed out that the specific expression of $T s s k 1 b$ is localized almost exclusively in the testes, which implicated a function related to reproduction and spermatogenesis [Hao et al., 2004]. Temporal expression studies further demonstrated that Tssk $1 b$ is expressed exclusively in the late stages of sperm maturation [Kueng et al., 1997]. The function of Tssk $1 b$ was confirmed in vivo by Xu et al. [2008] using chimeric mice to determine if a targeted deletion in Tssk $1 b$ and Tssk2 can be transmitted in the germline. Their experiments showed that the chimeric mice only produced wild-type offspring, even though sperm cells contained both the wild-type and the mutated allele. Further examination of the testis and epididymis in the chimera showed that only a low number of sperm could reach maturation [Xu et al., 2008]. In their study, $\mathrm{Xu}$ et al. [2008] suggested that the infertility is caused by haploinsufficiency. Although the exact mechanism of the pathological manifestation has not been studied, the functional implications of an allele deletion in this gene would seem to result in altered sperm morphology. In humans, a microdeletion encompassing TSSK1B may thus constitute a risk factor for male infertility.

So far, TSSK1B has been exclusively studied in animal models, and the functional implications have not been explored in humans. Here, we present what we consider

Interstitial Deletion of $5 \mathrm{q} 22.2 \mathrm{q} 23.1$

Including $A P C$ and TSSK $1 B$ the first case of correlation between a deleted copy of TSSK $1 B$ and asthenozoospermia, possibly confirming the link between gene and function in vivo.

\section{Conclusion}

This is one of the few cases describing a young adult with a large interstitial deletion in chromosome 5, with symptoms manifesting at an age when the patient can be tested for fertility. To the best of our knowledge, no patients with deletions in the 5q22.2 band have been examined for infertility, and only one case of a possible, yet unconfirmed inheritance of a TSSK1B deletion from a male carrier has been observed. Even the mildest developmental alterations observed in addition to the FAP phenotype should lead to further investigation of this chromosomal region beyond the $A P C$ gene, using the methods listed above to define any chromosomal alterations. We would also like to encourage the further study of the role of TSSK $1 B$ in male reproduction and its part in causing asthenozoospermia.

In addition, we demonstrate the usefulness of both MLPA and array CGH in clinical practice. MLPA might be considered as the first-line analysis, and if a deletion of the entire target gene is found, the results could be confirmed and the breakpoints localized by array CGH.

\section{Acknowledgment}

We are grateful to the family who provided the clinical information and gave permission to publish it.

\section{Statement of Ethics}

The patient signed informed consent forms for genetic testing and medical photography. The authors have no ethical conflicts to disclose.

\section{Disclosure Statement}

The authors declare no conflicts of interest.
Mol Syndromol 2018;9:235-240 DOI: $10.1159 / 000492516$ 


\section{References}

Bennett R, Karayiorgou M, Sobin C, Norwood T, Kay M: Identification of an interstitial deletion in an adult female with schizophrenia, mental retardation, and dysmorphic features: further support for a putative schizophreniasusceptibility locus at 5q21-23.1. Am J Hum Genet 61:1450-1454 (1997).

Bielke W, Blaschke R, Miescher G, Zürcher G, Andres A, Ziemiecki A: Characterization of a novel murine testis-specific serine/threonine kinase. Gene 139:235-239 (1994).

-Casper M, Petek E, Henn W, Niewald M, Schneider G, et al: Multidisciplinary treatment of desmoid tumours in Gardner's syndrome due to a large interstitial deletion of chromosome 5q. QJM 107:521-527 (2014).

- Cooper G, Coe B, Girirajan S, Rosenfeld J, Vu T, et al: A copy number variation morbidity map of developmental delay. Nat Genet 43:838846 (2011)

-Garcia-Miñaur S, Ramsay J, Grace E, Minns R, Myles L, FitzPatrick D: Interstitial deletion of the long arm of chromosome 5 in a boy with multiple congenital anomalies and mental retardation: molecular characterization of the deleted region to 5q22.3q23.3. Am J Med Genet A 132A:402-410 (2005).

- Hao Z, Jha KN, Kim YH, Vemuganti S, Westbrook VA, et al: Expression analysis of the human testis-specific serine/threonine kinase (TSSK) homologues. A TSSK member is present in the equatorial segment of human sperm. Mol Hum Reprod 10:433-444 (2004).

Heald B, Moran R, Milas M, Burke C, Eng C: Familial adenomatous polyposis in a patient with unexplained mental retardation. Nat Clin Pract Neurol 3:694-700 (2007).

ISCN 2016: An International System for Human Cytogenomic Nomenclature; McGowan-Jordan J, Simons A, Schmid M (eds). Cytogenet Genome Res 149:1-140 (2016).
Kadotani T, Katano T, Murakami M, Watanabe Y: A case of slight anemia with the long arm interstitial deletion of No. 5 chromosome (46, XY, 5q-). Proc Jpn Acad 55:300-304 (1979).

-Kinzler K, Nilbert M, Vogelstein B, Bryan T, Levy $\mathrm{D}$, et al: Identification of a gene located at chromosome $5 \mathrm{q} 21$ that is mutated in colorectal cancers. Science 251:1366-1370 (1991).

-Krausz C, Hoefsloot L, Simoni M, Tüttelmann F: EAA/EMQN best practice guidelines for molecular diagnosis of Y-chromosomal microdeletions: state-of-the-art 2013. Andrology 2: 5-19 (2014).

-Kueng P, Nikolova Z, Djonov V, Hemphill A, Rohrbach V, et al: A novel family of serine/ threonine kinases participating in spermiogenesis. J Cell Biol 139:1851-1859 (1997).

Lamb D, Song S, Chiba K, Ramasamy R: Recent advances in the genetics of testicular failure. Asian J Androl 18:350-355 (2016).

Lee S, Chae H, Park I, Kim M, Kim Y, et al: Genotype-phenotype correlation of a 5q22.3 deletion associated with craniofacial and limb defects. Gene 494:105-108 (2012).

Lindgren V, Bryke CR, Ozcelik T, Yang-Feng TL, Francke U: Phenotypic, cytogenetic, and molecular studies of three patients with constitutional deletions of chromosome 5 in the region of the gene for familial adenomatous polyposis. Am J Hum Genet 50:988-997 (1992).

O'Flynn O’Brien K, Varghese A, Agarwal A: The genetic causes of male factor infertility: a review. Fertil Steril 93:1-12 (2010).

- Ofner L, Raedle J, Windpassinger C, Schwarzbraun T, Kroisel P, et al: Phenotypic and molecular characterisation of a de novo $5 \mathrm{q}$ deletion that includes the APC gene. J Hum Genet 51:141-146 (2006).
Pilarski R, Brothman A, Benn P, Shulman Rosengren S: Attenuated familial adenomatous polyposis in a man with an interstitial deletion of chromosome arm 5q. Am J Med Genet 86: 321-324 (1999).

Raedle J, Friedl W, Engels H, Koenig R, Trojan J, Zeuzem S: A de novo deletion of chromosome $5 \mathrm{q}$ causing familial adenomatous polyposis, dysmorphic features, and mild mental retardation. Am J Gastroenterol 96:3016-3020 (2001).

Ray P, Toure A, Metzler-Guillemain C, Mitchell M, Arnoult C, Coutton C: Genetic abnormalities leading to qualitative defects of sperm morphology or function. Clin Genet 91:217232 (2017).

Rivera H, Simi P, Rossi S, Pardelli L, Di Paolo M: A constitutional 5q23 deletion. J Med Genet 27:267-268 (1990).

- Torrezan G, da Silva F, Krepischi A, dos Santos É, Rossi B, Carraro D: A novel SYBR-based duplex qPCR for the detection of gene dosage: detection of an $A P C$ large deletion in a familial adenomatous polyposis patient with an unusual phenotype. BMC Med Genet 13:55 (2012).

- Tzschach A, Krause-Plonka I, Menzel C, Kalscheuer V, Toennies H, et al: Molecular cytogenetic analysis of a de novo interstitial deletion of $5 \mathrm{q} 23.3 \mathrm{q} 31.2$ and its phenotypic consequences. Am J Med Genet A 140:496502 (2006).

Xu B, Hao Z, Jha K, Zhang Z, Urekar C, et al: Targeted deletion of Tssk 1 and 2 causes male infertility due to haploinsufficiency. Dev Biol 319:211-222 (2008).

Yamaguchi T, Koizumi K, Arai M, Tamura K, Iijima $\mathrm{T}$, et al: A large deletion of chromosome 5q22.1-22.2 associated with sparse type of familial adenomatous polyposis: report of a case. Jpn J Clin Oncol 44:1243-1247 (2014). 